\title{
$n \mathrm{D}$ システムのフィードバック補償による Practical 安定化 ${ }^{\dagger}$
}

徐

\author{
粒* ・池 田 貴 俊*・斉藤, 制 海*・阿 部, 健 一*
}

Feedback Practical-Stabilization of $n \mathrm{D}$ Systems

Li XU*, Takatoshi IKedA*, Osami SAIto* and Kenichi ABE*

\begin{abstract}
In many practical situations, the independent variables $i_{1}, \cdots, i_{n}$ of an $n \mathrm{D}$ signal $x\left(i_{1}, \cdots, i_{n}\right)$ are bounded spatial variables, except that perhaps one is the unbounded temporal variable. Taking this feature into account, Agathoklis and Bruton developed the concept of practical-BIBO stability for $n \mathrm{D}$ discrete systems, and showed that the conventional-BIBO stability conditions are too restrictive for many applications.

This paper deals with the problem of feedback practical-stabilization of $n \mathrm{D}$ discrete systems whose input and output signals are unbounded in, at most, one dimension. A constructive algorithm is first presented for solving Bezout equation over the ring of practically-stable rational functions. Then, a necessary and sufficient condition for an $n \mathrm{D}$ system to be practically-stabilizable is derived and the parametrization of all $n \mathrm{D}$ practically-stabilizing compensators is given. These results make it clear that the $n \mathrm{D}$ practical-stabilization problem can be essentially solved by using $1 \mathrm{D}$ approaches.
\end{abstract}

Key Words : practical-BIBO stability, $n \mathrm{D}$ systems, feedback, stabilization, Bezout equation

\section{1.はじめに}

多次元 $(n \mathrm{D})$ システムの重要な応用分野として, 画像処 理, 地震デー夕処理などがよく挙げられる. 一般に, こ のような分野で扱われる $n \mathrm{D}$ 信号 $x\left(i_{1}, \cdots, i_{n}\right)$ は, 独立 変数 $i_{1}, \cdots, i_{n}$ のうち,一つだけが時間変数, ほかは空間 変数という特徵がある. 加えて, 時間変数の変域には上 限がないのに対し, 空間変数はほとんど有界な変域に限 定されている11. 1D BIBO 安定の概念の直接的な拡張と して得られた $n \mathrm{D}$ システムの $\mathrm{BIBO}$ 安定性 ${ }^{2} に は$ は, 上に 述べた特徴が考慮されていない.すなわち, 従来の $n \mathrm{D}$ シ ステムの $\mathrm{BIBO}$ 安定性は, $n$. 個の独立変数に対して制限 条件はなく,すべての変数が同時に無限大になることが 許されるとの前提で, 定義されたものである.これに対 して, Agathoklis氏ら ${ }^{1)}$ は, $n$ 個の独立変数の中で, 一 つだけが無限になることができ, それ以外の変数の変域 はすべて有界であるとすることに基づく Practical-

†第 14 回 Dynamical System Theory シンポジウムで発表 $(1991 \cdot 12)$

* 豊橋技術科学大学 豊橋市天伯町字雲雀ヶ丘 1-1

* Toyohashi University of Technology, Toyohashi

(Received December 16, 1991)

(Revised April 23, 1992)
$\mathrm{BIBO}$ 安定性の概念を導入した.さらに, $n \mathrm{D}$ システムの Practical-BIBO 安定の判別が $n$ 個の $1 \mathrm{D}$ システムの安定 問題に帰着できることを示し, 従来の BIBO 安定性は多 くの実際の場合に対して厳し過ぎることを明らかにした.

本論文の目的は, $n \mathrm{D}$ システムのフィードバック安定 化問題をPractical 安定の視点から考察することであ る. Practical 安定とは閉ループ伝達関数行列のすべて の要素がPractical-BIBO安定であることである. Practical-BIBO 安定の概念は従来の BIBO 安定の場合 を包含するので, BIBO安定性は満足しないが Practical-BIBO 安定であるシステムが存在する11. この ことは, $n \mathrm{D}$ システムのフィードバック安定化問題への 従来のアプローチでは $n \mathrm{D}$ フィードバック Practical 安 定化問題は解決できないことを意味する，なぜなら，極 の個数が無限である難点を回避するために, 従来のアプ ローチでは, 与えられたプラントが分離型3) でなくても, 構成した閉ループ系を分離型にするということをその基 本としているからである ${ }^{4), 5)}$. 1D 多項式の積に分離できる $n \mathrm{D}$ 多項式において, おのおのの $1 \mathrm{D}$ 多項式が安定なら ば，もとの $n \mathrm{D}$ 多項式は必ず $n \mathrm{D} \mathrm{BIBO}$ 安定であると いう性質がある.この性質は従来の BIBO 安定なフィー ドバック系を設計するのに有用であるが, 明らかに 
11041992 年 9 月

(BIBO 安定ではないが) Practical-BIBO 安定であるシ ステムの設計には役立たない.したがって,より有効かつ 簡潔な設計法をみつける必要がある。

本論文では, 行列分解アプローチを用いて, $n \mathrm{D}$ システ ムのフィードバック Practical 安定化補償器の設計法を 提案する. それにより, $n \mathrm{D}$ システ公の Practical 安定化

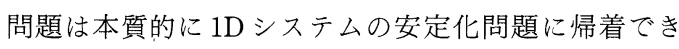
る.そこでは簡単な $n \mathrm{D}$ 多項式代数演算を除いてほとん ど1Dの手法で行えることが明らかにされる.以下，まず 準備として, $n \mathrm{D}$ システムの Practical-BIBO 安定の定 義, 条件抢よび従来の BIBO 安定性との比較を行う。つ ぎに, $n \mathrm{D}$ システムの Practical 安定な有理関数環 $\boldsymbol{H}$ に おける既約行列分解表現の定義と存在条件を与え，同時 に $\boldsymbol{H}$ 上の Bezout 方程式の解法を提案する.そしてこれ らの結果を用いて, Practical 安定化補償器の設計およ びパラメトリゼーションを行う. 最後に, 数値とシミュ レーション例で提案した方法の有効性を確認する.

本論文で使用する記号の一部をここで定義しておく。

$\boldsymbol{R}$ : 実数体, $\boldsymbol{C}$ : 複素数体, $A^{T}$ : 行列 $A$ の転置, $\boldsymbol{R}\left[z_{1}, \cdots, z_{n}\right]$ : 変数 $z_{1}, \cdots, z_{n}$ の実係数多項式環. $Z_{+}^{n}=\left\{\left(i_{1}, \cdots, i_{n}\right) \mid i_{1}, \cdots, i_{n}\right.$ は $\infty$ 含む正の整数 $\}$, $Z_{+}^{-n}=\left\{\left(i_{1}, \cdots, i_{n}\right) \mid i_{1}, \cdots, i_{n}\right.$ は $\infty$ を含む正の整数で, た だし二つ以上が同時にのになることができない＼$}$

$$
\begin{aligned}
& \bar{U}=\{z \in C|| z \mid \leq 1\} \\
& \bar{U}^{n}=\left\{\left(z_{1}, \cdots, z_{n}\right) \in C^{n}|| z_{k} \mid \in \bar{U}, k=1, \cdots, n\right\} \\
& U^{n}=\left\{\left(z_{1}, \cdots, z_{n}\right) \in C^{n}|| z_{k} \mid<1, k=1, \cdots, n\right\} \\
& T^{n}=\left\{\left(z_{1}, \cdots, z_{n}\right) \in C^{n}|| z_{k} \mid=1, k=1, \cdots, n\right\}
\end{aligned}
$$

\section{2. $n \mathrm{D}$ システムの Practical-BIBO 安定性}

入出力関係がつぎのような $n \mathrm{D}$ 畳み込みにより表現さ れる 1 入力 1 出力線形時不変 causal $n \mathrm{D}$ 離散システム を考える。

$$
\begin{aligned}
y\left(i_{1}, \cdots, i_{n}\right)= & \sum_{k_{1}=0}^{i_{1}} \cdots \sum_{k_{n}=0}^{i_{n}} \\
& g\left(i_{1}-k_{1}, \cdots, i_{n}-k_{n}\right) u\left(k_{1}, \cdots, k_{n}\right)
\end{aligned}
$$

ただし, $u\left(i_{1}, \cdots, i_{n}\right)$ は入力, $y\left(i_{1}, \cdots, i_{n}\right)$ は出力, $g\left(i_{1}\right.$, $\left.\cdots, i_{n}\right)$ はインパルス応答である. $n \mathrm{D} Z$-変換より, この システムの伝達関数が $(2)$ 式のように求められる.

$$
\begin{aligned}
g\left(z_{1}, \cdots, z_{n}\right) & =\mathscr{Z}\left[g\left(i_{1}, \cdots, i_{n}\right)\right] \\
& =\sum_{i_{1}=0}^{\infty} \cdots \sum_{i_{n}=0}^{\infty} g\left(i_{1}, \cdots, i_{n}\right) z_{1}^{i_{1}} \cdots z_{n}^{i_{n}}(2)
\end{aligned}
$$

ただし， $z_{i}, i=1, \cdots, n$ は単位遅れ演算子である.

(1) 式の $n \mathrm{D}$ 離散システムの $\mathrm{BIBO}$ 安定の定義はつ ぎのように与えられている.

【定義 2.1 2),6) $^{2,1}$ 式の $n \mathrm{D}$ 離散システムにおいて, あ
第 28 巻 第 9 号

る有限の実数 $M$ について,

$$
\left|u\left(i_{1}, \cdots, i_{n}\right)\right| \leq M<\infty \quad \forall\left(i_{1}, \cdots, i_{n}\right) \in Z_{+}^{n}
$$

を満たすすべての入力 $u\left(i_{1}, \cdots, i_{n}\right)$ に対し, 出力 $y\left(i_{1}\right.$, $\left.\cdots, i_{n}\right)$ もまた有界，すなわち

$$
\left|y\left(i_{1}, \cdots, i_{n}\right)\right| \leq L<\infty
$$

を満足する有限の実数 $L$ が実在するとき，またそのとき に限り, システム $(1)$ は BIBO 安定であるという.

一方, Practical-BIBO 安定はつぎのように定義される.

【定義 2.2】 (1) 式の $n \mathrm{D}$ 離散システムにおいて, ある 有限の実数 $M$ について,

$$
\left|u\left(i_{1}, \cdots, i_{n}\right)\right| \leq M<\infty \quad \forall\left(i_{1}, \cdots, i_{n}\right) \in Z_{+}^{-n}
$$

を満たすすべての入力 $u\left(i_{1}, \cdots, i_{n}\right)$ に対する出力 $y\left(i_{1}\right.$, $\left.\cdots, i_{n}\right)$ に関し

$$
\left|y\left(i_{1}, \cdots, i_{n}\right)\right| \leq L<\infty
$$

を満足する有限の実数 $L$ が存在するとき，またそのとき に限り, システム (1)は Practical-BIBO 安定であると いう.

両者の相違は, 従来の BIBO 安定の定義ではすべての 独立変数が同時に無限大になることができるのに対し， Practical-BIBO 安定の定義では, 二つ以上の独立変数が 同時に無限大にならないところにある.

よく知られているように, システムのインパルス応答 がつぎの関係式を満たすことが従来の BIBO 安定のた めの必要十分条件である2),6).

$$
\sum_{i_{1}=0}^{\infty} \sum_{i_{2}=0}^{\infty} \cdots \sum_{i_{n}=0}^{\infty}\left|g\left(i_{1}, i_{2}, \cdots, i_{n}\right)\right|<\infty
$$

これに対し, Agathoklis 氏らは Practical-BIBO安定 についてつぎの結果を与えた.

《定理 2.1》》1 $n \mathrm{D}$ 離散システム ( 1) が Practical-BIBO 安定であるための必要十分条件は, つぎの $n$ 個の不等式 が成立することである。

$$
\begin{array}{r}
\sum_{i_{1}=0}^{N_{1}} \sum_{i_{2}=0}^{N_{2}} \cdots \sum_{i_{k}=0}^{N_{k}=\infty} \cdots \sum_{i_{n}=0}^{N_{n}}\left|g\left(i_{1}, i_{2}, \cdots, i_{k}, \cdots, i_{n}\right)\right|<\infty \\
k=1,2, \cdots, n
\end{array}
$$

ただし, $N_{1}, N_{2}, \cdots, N_{k-1}, N_{k+1}, \cdots, N_{n}$ はすべて有限の整 数である.

$n \mathrm{D}$ 離散システム $(1)$ の伝達関数 $g\left(z_{1}, \cdots, z_{n}\right)$ はつぎ のように $n \mathrm{D}$ causal 有理関数で表わすことができる.

$$
g\left(z_{1}, \cdots, z_{n}\right)=\frac{n\left(z_{1}, \cdots, z_{n}\right)}{d\left(z_{1}, \cdots, z_{n}\right)}
$$

ただし, $n, d \in \boldsymbol{R}\left[z_{1}, \cdots, z_{n}\right], d(0, \cdots, 0) \neq 0$. PracticalBIBO 安定と伝達関数の特異点の関係は, 定理 2.2 より 明らかにされている。

《定理 2.2》1) $n \mathrm{D}$ 離散システム $(9)$ が Practical-BIBO 安定であるための必要十分条件は

$$
d\left(0, \cdots, 0, z_{k}, 0, \cdots, 0\right) \neq 0
$$


$\forall z_{k} \in \bar{U}, k=1,2, \cdots, n$

であることである.

$n \mathrm{D}$ システム $(9)$ が, $T^{2}\left(n=2\right.$ の場合7) $\left.{ }^{7}\right)$ または $\bar{U}^{n}$ $-U^{n}\left(n>2\right.$ の場合 $\left.{ }^{8)}\right)$ において第二種の特異点(nonessential singularity of the second kind ${ }^{9)}$ )をもたない ならば, BIBO 安定であるための必要十分条件は,

$$
d\left(z_{1}, \cdots, z_{n}\right) \neq 0 \quad \forall\left(z_{1}, \cdots, z_{n}\right) \in \bar{U}^{n}
$$

であることが知られている。

条件 (10)と（11）を比較すれば，(10)のほうがずっと緩 いことがわかる，逆にみれば，従来の BIBO 安定の定義 は, 多くの実際の場合に対して厳しすぎるともいえる. また, 定理 2.2 より, $n \mathrm{D}$ システムの Practical-BIBO 安 定性のテストは条件 (10)の $n$ 個の $1 \mathrm{D}$ 多項式の安定性を 別々に判定すればよいことも明らかであろう。

\section{3. $n \mathrm{D}$ システムの Practical 安定な有理関数環上 の既約行列分解表現}

まず, $n \mathrm{D}$ causal 有理関数環 $\boldsymbol{G}$ と, $n \mathrm{D}$ Practical 安定な 有理関数環 $\boldsymbol{H}$ をつぎのように定義する.

$$
\begin{gathered}
\boldsymbol{G}=\left\{n / d \mid n, d \in \boldsymbol{R}\left[z_{1}, \cdots, z_{n}\right], d(0, \cdots, 0) \neq 0\right\} \\
\boldsymbol{H}=\left\{n / d \in \boldsymbol{G} \mid d\left(0, \cdots, 0, z_{k}, 0, \cdots, 0\right) \neq 0\right. \\
\left.\forall z_{k} \in \bar{U}, k=1,2, \cdots, n\right\}
\end{gathered}
$$

また，

$$
\begin{aligned}
& \boldsymbol{I}=\left\{h \in \boldsymbol{H} \mid h^{-1} \in \boldsymbol{G}\right\} \\
& \boldsymbol{J}=\left\{h \in \boldsymbol{H} \mid h^{-1} \in \boldsymbol{H}\right\}
\end{aligned}
$$

とする. $\boldsymbol{M}(*)$ で集合*の要素で構成された行列の集合 を表わす. $\boldsymbol{M}(\boldsymbol{H})$ の行列について，それが正方で，しかも その行列式が $\boldsymbol{I}(\boldsymbol{J})$ に属すれば, $\boldsymbol{G}$-unimodular ( $\boldsymbol{H}$-unimodular)であるという。 $P \in \boldsymbol{M}(\boldsymbol{G})$ に対し,$P=N_{p} D_{p}^{-1}$ なる $N_{p} \in \boldsymbol{M}(\boldsymbol{H})$ と $\boldsymbol{G}$-unimodularである $D_{p}$ が存在す るとき, $N_{p} D_{p}^{-1}$ を $P$ の $(\{\boldsymbol{G}, \boldsymbol{H}, \boldsymbol{I}, \boldsymbol{J}\}$ における $)$ 右行列 分解表現という。

【定義 3.1】右行列分解表現 $N_{p} D_{p}^{-1}$ に対して, Bezout 方程式 (12) を満たす $U, V \in \boldsymbol{M}(\boldsymbol{H})$ が存在するとき,また そのときに限り， $N_{p}$ と $D_{p}$ は $\boldsymbol{H}$ において右既約である といい， $N_{p} D_{p}^{-1}$ を $\boldsymbol{H}$ 上の右既約行列分解表現という.

$$
U D_{p}+V N_{p}=I
$$

以上の定義は，左についても同様に行われる．任意の $P \in \boldsymbol{M}(\boldsymbol{G})$ には, $N_{p}, D_{p} \in \boldsymbol{M}\left(\boldsymbol{R}\left[z_{1}, \cdots, z_{n}\right]\right) \subset \boldsymbol{M}(\boldsymbol{H})$ な る右行列分解表現 $P=N_{p} D_{p}^{-1}$ が必ず存在するが, それが $\boldsymbol{H}$ において右既約とは限らない. それで, 本論文の基本的 な結果として, $P$ の $\boldsymbol{H}$ 上の (右) 既約行列分解表現の存在 条件を与える.以下,一般性を失わずに, $N_{p}, D_{p} \in \boldsymbol{M}\left(\boldsymbol{R}\left[z_{1}\right.\right.$, $\left.\left.\cdots, z_{n}\right]\right)$ と仮定する。 また， $\mathscr{I}_{k}$ で行列

$$
\left[D_{p}^{T}\left(0, \cdots, z_{k}, \cdots, 0\right) N_{p}^{T}\left(0, \cdots, z_{k}, \cdots, 0\right)\right]^{T}
$$

のすべての最大次数の小行列式より生成されたイデアル
を表わす。V( $\left.\mathscr{I}_{k}\right) て ゙ \mathscr{I}_{k}$ の algebraic variety，つまり， それらの最大次数の小行列式の最大共通因子の零点の集 合を表わす.ただし $, k=1,2, \cdots, n$.すると,つぎの結果が 得られる。

《定理 3. 1》 $N_{p}, D_{p} \in \boldsymbol{M}\left(\boldsymbol{R}\left[z_{1}, \cdots, z_{n}\right]\right)$ なる右行列分解表 現 $N_{p} D_{p}^{-1}$ に対して, $D_{p}$ と $N_{p}$ が $\boldsymbol{H}$ において右既約である 必要十分条件は

$$
V\left(\mathscr{I}_{k}\right) \cap \bar{U}=\phi, \quad k=1,2, \cdots, n
$$

であることである。

（証明） 方程式(12)の可解性は, 明らかに,つぎの $n \mathrm{D}$ 多 項式行列方程式を満たす $X, Y, \Phi$ が存在することと等 価である。

$$
X D_{p}+Y N_{p}=\Phi
$$
ただし， $X, Y, \Phi \in \boldsymbol{M}\left(\boldsymbol{R}\left[z_{1}, \cdots, z_{n}\right]\right)$ ，加つ $\operatorname{det} \Phi \in \boldsymbol{J}$. 必要性: Cauchy-Binet 定理より, $z_{k}^{0} \in \mathscr{V}\left(\mathscr{I}_{k}\right)$ は

$$
\begin{aligned}
\operatorname{det} & \Phi\left(0, \cdots, z_{k}, \cdots, 0\right) \\
= & \operatorname{det}\left\{\left[X\left(0, \cdots, z_{k}, \cdots, 0\right) Y\left(0, \cdots, z_{k}, \cdots, 0\right)\right]\right. \\
& {\left.\left[\begin{array}{l}
D_{p}\left(0, \cdots, z_{k}, \cdots, 0\right) \\
N_{p}\left(0, \cdots, z_{k}, \cdots, 0\right)
\end{array}\right]\right\} }
\end{aligned}
$$

の零点でもある.よって, $z_{k}^{0} \in \bar{U}$ ならば, $\operatorname{det} \Phi \notin \boldsymbol{J}, つ$ まり，方程式(14)は成立しない。

十分性：条件(13)が成り立つとすると，1Dの手法で, $\boldsymbol{R}\left[z_{k}\right]$ においてつぎの $1 \mathrm{D}$ 多項式行列方程式の解を求め ることができる10).

$$
\begin{aligned}
& \tilde{X}_{k}\left(z_{k}\right) D_{p}\left(0, \cdots, z_{k}, \cdots, 0\right) \\
& \quad+\tilde{Y}_{k}\left(z_{k}\right) N_{p}\left(0, \cdots, z_{k}, \cdots, 0\right)=\tilde{\Phi}_{k}\left(z_{k}\right)
\end{aligned}
$$

ここで,

$$
\operatorname{det} \tilde{\Phi}_{k}\left(z_{k}\right) \neq 0 \quad \forall z_{k} \in \bar{U}, \quad k=1,2, \cdots, n
$$
これらの方程式の一般解は，つぎのように表わされる。

$$
\left\{\begin{array}{l}
\bar{X}_{k}\left(z_{k}\right)=\tilde{X}_{k}\left(z_{k}\right)+R_{k}\left(z_{k}\right) \tilde{N}_{k}\left(z_{k}\right) \\
\bar{Y}_{k}\left(z_{k}\right)=\tilde{Y}_{k}\left(z_{k}\right)-R_{k}\left(z_{k}\right) \tilde{D}_{k}\left(z_{k}\right)
\end{array}\right.
$$

ただし, $R_{k}\left(z_{k}\right)$ は $z_{k}$ に関する任意の $1 \mathrm{D}$ 多項式行列であ り, $\tilde{D}_{k}\left(z_{k}\right), \tilde{N}_{k}\left(z_{k}\right)$ は次式を満足し, しかも, $\boldsymbol{R}\left[z_{k}\right]$ にお いて左既約である。

$$
\begin{aligned}
& \tilde{D}_{k}^{-1}\left(z_{k}\right) \tilde{N}_{k}\left(z_{k}\right) \\
& \quad=N_{p}\left(0, \cdots, z_{k}, \cdots, 0\right) D_{p}^{-1}\left(0, \cdots, z_{k}, \cdots 0\right)
\end{aligned}
$$

$E_{k}\left(z_{k}\right)$ を $D_{p}\left(0, \cdots, z_{k}, \cdots, 0\right)$ と $N_{p}\left(0, \cdots, z_{k}, \cdots, 0\right)$ の 最大右共通因子行列とすれば,

$\operatorname{det} D_{p}\left(0, \cdots, z_{k}, \cdots, 0\right)=\operatorname{det} \tilde{D}_{k}\left(z_{k}\right) \operatorname{det} E_{k}\left(z_{k}\right)$

が成り立つ。また, $\operatorname{det} D_{p} \in \boldsymbol{I}, つ ま り, \operatorname{det} D_{p}(0, \cdots, 0)$ キ0なので,

$\operatorname{det} \widetilde{D}_{k}(0) \neq 0$

である、したがって，

$$
R_{k}\left(z_{k}\right)=\tilde{Y}_{k}(0) \tilde{D}_{k}^{-1}(0) \triangleq R_{k} \in \boldsymbol{R}
$$


と設定することができる， $R_{k}$ を(18) 式に代入し, また方 程式(16)を用いれば，つぎの結果が明らかである。

$$
\operatorname{det} \bar{X}_{k}(0)=\operatorname{det}\left\{\widetilde{\Phi}_{k}(0) \dot{D}_{p}^{-1}(0, \cdots, 0)\right\} \neq 0
$$

$$
\bar{Y}_{k}(0)=0
$$

一方, $\bar{X}_{k}\left(z_{k}\right)$ はつぎのように変数 $z_{k}$ を含む項の部分と 定数項の部分を分離して表現することができる.

$$
\bar{X}_{k}\left(z_{k}\right) \triangleq \widehat{X}_{k}\left(z_{k}\right)+\bar{X}_{k}(0)
$$

ただし，

$$
\widehat{X}_{k}(0)=0
$$

(23) 式より, $\bar{X}_{k}^{-1}(0)$ が存在する. そして, $\bar{X}_{k}\left(z_{k}\right), \bar{Y}_{k}\left(z_{k}\right)$ を方程式(16)に代入して，また左から $\bar{X}_{k}^{-1}(0)$ をかける と,つぎの結果が得られる。

$$
\begin{aligned}
& X_{k}\left(z_{k}\right) D_{p}\left(0, \cdots, z_{k}, \cdots, 0\right)+ \\
& \quad+Y_{k}\left(z_{k}\right) N_{p}\left(0, \cdots, z_{k}, \cdots, 0\right)=\Phi_{k}\left(z_{k}\right)
\end{aligned}
$$
ただし，

$$
\begin{aligned}
X_{k}\left(z_{k}\right) & =\bar{X}_{k}^{-1}(0) \bar{X}_{k}\left(z_{k}\right)=\bar{X}_{k}^{-1}(0) \hat{X}_{k}\left(z_{k}\right)+I \\
& \triangleq X_{k}^{\prime}\left(z_{k}\right)+I \\
Y_{k}\left(z_{k}\right) & =\bar{X}_{k}^{-1}(0) \bar{Y}_{k}\left(z_{k}\right) \\
\Phi_{k}\left(z_{k}\right) & =\bar{X}_{k}^{-1}(0) \tilde{\Phi}_{k}\left(z_{k}\right)
\end{aligned}
$$

また，ここで,

$$
\begin{aligned}
& X_{k}^{\prime}(0)=0 \\
& Y_{k}(0)=0 \\
& \operatorname{det} \Phi_{k}\left(z_{k}\right) \neq 0 \quad \forall z_{k} \in \bar{U}
\end{aligned}
$$

以上の結果に基づいて, 方程式(14)の解 $X\left(z_{1}, \cdots, z_{n}\right)$, $Y\left(z_{1}, \cdots, z_{n}\right)$ をつぎのように構成できる.

$$
\begin{aligned}
& X\left(z_{1}, \cdots, z_{n}\right)=\sum_{k=1}^{n} X_{k}^{\prime}\left(z_{k}\right)+I \\
& Y\left(z_{1}, \cdots, z_{n}\right)=\sum_{k=1}^{n} Y_{k}\left(z_{k}\right)
\end{aligned}
$$

ただし， $\Phi\left(z_{1}, \cdots, z_{n}\right)$ はつぎの式より計算される.

$$
\begin{aligned}
\Phi\left(z_{1}, \cdots, z_{n}\right)= & \left\{\sum_{k=1}^{n} X_{k}^{\prime}\left(z_{k}\right)+I\right\} D_{p}\left(z_{1}, \cdots, z_{n}\right) \\
& +\left\{\sum_{k=1}^{n} Y_{k}\left(z_{k}\right)\right\} N_{p}\left(z_{1}, \cdots, z_{n}\right)
\end{aligned}
$$

しかも，（27）～(35) 式よりつぎの関係式を満たす.

$$
\begin{aligned}
& \Phi\left(0, \cdots, z_{k}, \cdots, 0\right) \\
&=\left\{\sum_{\substack{j=1 \\
j \neq k}}^{n} X_{j}^{\prime}(0)+X_{k}^{\prime}\left(z_{k}\right)+I\right\} D_{p}\left(0, \cdots, z_{k}, \cdots, 0\right) \\
&+\left\{\sum_{\substack{j=1 \\
j \neq k}}^{n} Y_{j}(0)+Y_{k}\left(z_{k}\right)\right\} N_{p}\left(0, \cdots, z_{k}, \cdots, 0\right) \\
&= X_{k}\left(z_{k}\right) D_{p}\left(0, \cdots, z_{k}, \cdots, 0\right) \\
&+Y_{k}\left(z_{k}\right) N_{p}\left(0, \cdots, z_{k}, \cdots, 0\right) \\
&= \Phi_{k}\left(z_{k}\right)
\end{aligned}
$$

よって,

$\operatorname{det} \Phi\left(0, \cdots, z_{k}, \cdots, 0\right)=\operatorname{det} \Phi_{k}\left(z_{k}\right) \neq 0$

$$
\forall z_{k} \in \bar{U}, \quad k=1, \cdots, n
$$

つまり, det $\Phi \in \boldsymbol{J}$. したがって, 方程式(12)の解 $U, V$ $\in \boldsymbol{M}(\boldsymbol{H})$ はただちに

$$
\left\{\begin{array}{l}
U=\Phi^{-1} X \\
V=\Phi^{-1} Y
\end{array}\right.
$$

と構成できる.

定理 3.1 の条件 (13) は, 各 $k$ について, すべての $z_{j}(j$ $\neq k)$ を 0 とした多項式行列 $D_{p}\left(0, \cdots, z_{k}, \cdots, 0\right)$ と $N_{p}(0$, $\left.\cdots, z_{k}, \cdots, 0\right)$ とが安定な $1 \mathrm{D}$ 有理関数環上で右既約であ ることと等価である.

$\boldsymbol{H}$ 上の左と右既約行列分解表現に基づいて, つぎの $\boldsymbol{H}$ 上の重既約行列分解表現も得られる.

《定理 3.2》 $N_{p} D_{p}^{-1}$ と $\tilde{D}_{p}^{-1} \tilde{N}_{p}$ をそれぞれ $P \in \boldsymbol{M}(\boldsymbol{G})$ の $\boldsymbol{H}$ 上の右と左既約行列分解表現とする．このとき，つぎの $\boldsymbol{H}$ 上の重既約分解表現を満たす $U, V, \tilde{U}, \tilde{V} \in \boldsymbol{M}(\boldsymbol{H})$ が存在する。

$$
\left[\begin{array}{cc}
U & V \\
-\widetilde{N}_{p} & \widetilde{D}_{p}
\end{array}\right]\left[\begin{array}{cc}
D_{p} & -\tilde{V} \\
N_{p} & \tilde{U}
\end{array}\right]=\left[\begin{array}{cc}
I & 0 \\
0 & I
\end{array}\right]
$$

（証明） $1 \mathrm{D}$ の場合と同様に証明できる ${ }^{11), 12)}$.

\section{4. $n \mathrm{D}$ 多入力多出カシステムのフィードバック Practical 安定化補償}

本章では, $n \mathrm{D}$ システムの Practical 安定の意味での フィードバック安定化補償問題は 3 章の結果より本質的 に1Dの手法で, 簡単に解決できることを示そう.

Fig. 1 に示されている $n \mathrm{D}$ 多入力多出力フィードバッ クシステムを考える.ここで, $P \in \boldsymbol{M}(\boldsymbol{G})$ はプラントで, $P$ の $\boldsymbol{H}$ 上の右既約行列分解表現を

$$
P=N_{p} D_{p}^{-1}
$$

とする.ただし, $N_{p}, D_{p} \in \boldsymbol{M}\left(\boldsymbol{R}\left[z_{1}, \cdots, z_{n}\right]\right) . C \in \boldsymbol{M}(\boldsymbol{G})$ は コントローラで, その左行列分解表現を

$$
C=D_{c}^{-1}\left[\begin{array}{ll}
N_{c 1} & N_{c 2}
\end{array}\right]
$$

とする(波1). ここで, $D_{c}, N_{c 1}, N_{c 2} \in \boldsymbol{M}(\boldsymbol{H})$ および $D_{c}$ と $N_{c 2}$ は $\boldsymbol{H}$ において左既約であるとする。 そして，

$$
y=\left[\begin{array}{l}
y_{1} \\
y_{2}
\end{array}\right], \quad u=\left[\begin{array}{l}
u_{1} \\
u_{2} \\
u_{3}
\end{array}\right]
$$

とすると，つぎの関係式が得られる。

$$
y=H_{y u} u
$$

ただし，

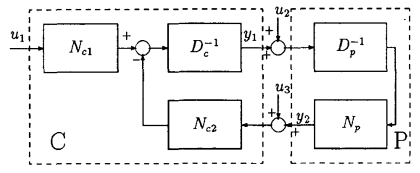

Fig. $1 n$ D feedback control system

（注 1 ）安定化補償のみの議論では 1 自由度制御で十分である が,つぎの 5 章での議論のため, このような 2 自由度制御系 表現とした。 


$$
\begin{aligned}
& H_{y u}=\left[\begin{array}{ccc}
D_{p} \Delta^{-1} N_{c 1} & -I+D_{p} \Delta^{-1} D_{c} & D_{p} \Delta^{-1} N_{c 2} \\
N_{p} \Delta^{-1} N_{c 1} & N_{p} \Delta^{-1} D_{c} & N_{p} \Delta^{-1} N_{c 2}
\end{array}\right] \\
& \Delta=N_{c 2} N_{p}+D_{c} D_{p}
\end{aligned}
$$

このとき, $\operatorname{det} \Delta \neq 0$, かつ $H_{y u} \in \boldsymbol{M}(\boldsymbol{H})$ ならば, Fig. 1 の フィードバックシステムがPractical安定であるという。 また, $H_{y u} \in \boldsymbol{M}(\boldsymbol{H})$ なる $C \in \boldsymbol{M}(\boldsymbol{G})$ が存在すれば, $P$ は Practical 可安定であるといい, $C$ をこのシステムの Practical 安定化補償器という.

[命題 4.1] Fig.1のフィードバックシステムが Practical安定であるための必要十分条件は， $\Delta$ が $\boldsymbol{H}$ unimodularであることである。

（証明）十分性は明らか。必要性は，文献 11)，12）と同 じ方法で， $\boldsymbol{H}$ において， $D_{p}, N_{p}$ が右既約， $D_{c}, N_{c 2}$ が左 既約であるという仮定条件を用いれば，証明できる。

《定理 4. 1》 $n \mathrm{D}$ プラント $P=N_{p} D_{p}^{-1} \in \boldsymbol{M}(\boldsymbol{G})$ が causal コントローラによって Practical 可安定であるための必 要十分条件は $D_{p}$ と $N_{p}$ が $\boldsymbol{H}$ において右既約であるこ とである。

(証明) $D_{p}$ と $N_{p}$ が $\boldsymbol{H}$ に扑て右既約であるとすると， 定理 3.1 の十分性の証明で示された方法で，方程式(12) を満たす $U, V \in \boldsymbol{M}(\boldsymbol{H})$ が求められる。しかも，(39), (34)，（31），(38)式より

$$
\operatorname{det} U(0, \cdots, 0) \neq 0
$$

よって, $C=U^{-1} V \in \boldsymbol{M}(\boldsymbol{G})$. したがって, 単位行列 $I$ が $\boldsymbol{H}$-unimodular であることと命題 4.1 より, 十分性が成 り立つ.

逆に, 命題 4.1 によって, $C=X^{-1} Y=X^{-1}\left[\begin{array}{ll}Y_{1} & Y_{2}\end{array}\right] \in$ $\boldsymbol{M}(\boldsymbol{G})$ が $P$ の Practical 安定化補償器であれば,

$$
X D_{p}+Y_{2} N_{p}=\Phi
$$

が成立する.ただし， $\Phi$ は $\boldsymbol{H}$-unimodularである. (52)式は 左から $\Phi^{-1}$ をかけると,

$$
\left(\Phi^{-1} X\right) D_{p}+\left(\Phi^{-1} Y_{2}\right) N_{p}=I
$$

となり， $D_{p}$ と $N_{p}$ が $\boldsymbol{H}$ において右既約であることを示 している.

以上の結果に基づいて, Practical 安定化補償器のパ ラメトリゼーションはつぎのようにできる.

《定理 4.2》 $N_{p} D_{p}^{-1}$ と $\tilde{D}_{p}^{-1} \tilde{N}_{p}$ をそれぞれプラント $P$ $\in \boldsymbol{M}(\boldsymbol{G})$ の $\boldsymbol{H}$ 上の右と左既約行列分解表現とする.また, $U, V \in \boldsymbol{M}(\boldsymbol{H})$ が方程式 $U D_{p}+V N_{p}=I$ を満足するとす る.このとき, $P$ のすべての Practical 安定化補償器 $C$ $\in \boldsymbol{M}(\boldsymbol{G})$ のクラスはつぎのように与えられる.

$$
\begin{aligned}
C \in\left\{\left(U+S \tilde{N}_{p}\right)^{-1}[Q \quad\right. & \left.V-S \tilde{D}_{p}\right] \mid \\
& \left.Q, S \in \boldsymbol{M}(\boldsymbol{H}), \operatorname{det}\left(U+S \tilde{N}_{p}\right) \in \boldsymbol{I}\right\}
\end{aligned}
$$

$$
\left[\begin{array}{lll}
D_{p} Q & D_{p}\left(U+S \tilde{N}_{p}\right)-I & D_{p}\left(V-S \tilde{D}_{p}\right) \\
N_{p} Q & N_{p}\left(U+S \tilde{N}_{p}\right) & N_{p}\left(V-S \tilde{D}_{p}\right)
\end{array}\right]
$$

となっている.

（証明）十分性は明らか. 必要性は文献 13),4）と同じ方法 で, 定理 3.1 と定理 4.1 の結果を用いて証明できる. [

\section{5. 数值とシミュレーション例}

ここで, 数值とシミュレーション例をとおして, 提案 した設計法の有効性を確認する。

〔例 5.1〕 伝達関数行列がつぎのような $\boldsymbol{R}\left[z_{1}, z_{2}\right]$ にお ける右因子既約 (factor coprime) ${ }^{9)}$ 行列分解表現で表わ される 2D MIMO システムを考える。

$$
P\left(z_{1}, z_{2}\right)=N_{p}\left(z_{1}, z_{2}\right) D_{p}^{-1}\left(z_{1}, z_{2}\right)
$$

ただし，

$$
\begin{aligned}
& D_{p}\left(z_{1}, z_{2}\right) \\
& \quad=\left[\begin{array}{cc}
\frac{\left(2 z_{1}-1\right)\left(2 z_{1}-5\right)}{16} & -\frac{\left(2 z_{2}-1\right)\left(2 z_{1}-5\right) z_{2}}{2} \\
0 & 2\left(6 z_{1}+8 z_{2}-7\right)
\end{array}\right] \\
& N_{p}\left(z_{1}, z_{2}\right) \\
& \quad=\left[\begin{array}{cc}
\frac{\left(2 z_{1}-1\right) z_{2}}{16} & \frac{8 z_{1}-2 z_{2}^{3}+z_{2}^{2}-4}{2} \\
\frac{\left(2 z_{1}-5\right)^{2}}{16} & -\frac{\left(4 z_{1} z_{2}-2 z_{1}-18 z_{2}-3\right) z_{2}}{2}
\end{array}\right]
\end{aligned}
$$

文献 5)の方法で, $\left(z_{1}, z_{2}\right)=(1 / 2,1 / 2) \in \bar{U}^{2}$ が行列 $\left[D_{p}^{T}\left(z_{1}, z_{2}\right) \quad N_{p}^{T}\left(z_{1}, z_{2}\right)\right]^{T}$ のすべての $2 \times 2$ 小行列式の共 通零点であることがわかる。したがって， $P$ は従来の BIBO 安定の意味では不可安定である ${ }^{4) 5}$.

一方, $D_{p}\left(z_{1}, 0\right)$ と $N_{p}\left(z_{1}, 0\right)$ の最大右共通因子行列 $G\left(z_{1}\right)$ は

$$
G\left(z_{1}\right)=\left[\begin{array}{cc}
\frac{1}{2}\left(2 z_{1}-5\right) & 0 \\
0 & 1
\end{array}\right]
$$

であることから, $V\left(\mathscr{I}_{1}\right) \cap \bar{U}=\{5 / 2\} \cap \bar{U}=\phi$ が導かれ る.また, $D_{p}\left(0, z_{2}\right)$ と $N_{p}\left(0, z_{2}\right)$ が $\boldsymbol{R}\left[z_{2}\right]$ に抢いて既約,

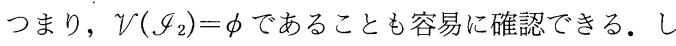
たがって, 定理 4.1 と定理 3.1 にっっ, $P$ は Practical 可安定である.

$P$ の Practical 安定化補償器はつぎの手順で構成でき る.まず, 方程式 (16)の解がつぎのように求められる.

$$
\begin{aligned}
& \tilde{X}_{1}\left(z_{1}\right)=\left[\begin{array}{cc}
2 & 0 \\
0 & -\frac{1}{8}
\end{array}\right] \quad \tilde{Y}_{1}\left(z_{1}\right)=\left[\begin{array}{cc}
0 & -2 \\
\frac{8}{3} & 0
\end{array}\right] \\
& \widetilde{\Phi}_{1}\left(z_{1}\right)=\left[\begin{array}{cc}
\frac{1}{2}\left(2 z_{1}-5\right) & 0 \\
0 & 1
\end{array}\right]
\end{aligned}
$$


11081992 年 9 月

$$
\begin{aligned}
& \tilde{X}_{2}\left(z_{2}\right)=\left[\begin{array}{cc}
\frac{4}{5}\left(2 z_{2}^{3}-z_{2}^{2}+4\right) & 0 \\
-\frac{1}{10} z_{2} & 0
\end{array}\right] \\
& \tilde{Y}_{2}\left(z_{2}\right)=\left[\begin{array}{cc}
4 z_{2}\left(2 z_{2}-1\right) & 0 \\
-\frac{1}{2} & 0
\end{array}\right] \quad \tilde{\Phi}_{2}\left(z_{2}\right)=\left[\begin{array}{ll}
1 & 0 \\
0 & 1
\end{array}\right]
\end{aligned}
$$

ここで, 明らかに, $\operatorname{det} \widetilde{\Phi}_{1}(z)=(2 z-5) / 2 \neq 0, \operatorname{det} \widetilde{\Phi}_{2}(z)$ $=1 \neq 0, \forall z \in \bar{U}$.

つぎに，(19) 式を満たす $\tilde{D}_{1}, \tilde{N}_{1}$ と $\tilde{D}_{2}, \tilde{N}_{2}$ を求める.

$$
\begin{aligned}
& \tilde{D}_{1}\left(z_{1}\right)=\left[\begin{array}{cc}
\frac{3}{4}\left(6 z_{1}-7\right) & 0 \\
0 & -\frac{1}{4}\left(2 z_{1}-1\right)
\end{array}\right] \\
& \tilde{N}_{1}\left(z_{1}\right)=\left[\begin{array}{cc}
0 & \frac{3}{4}\left(2 z_{1}-1\right) \\
-\frac{1}{4}\left(2 z_{1}-5\right) & 0
\end{array}\right] \\
& \tilde{D}_{2}\left(z_{2}\right)=\left[\begin{array}{cc}
-\left(8 z_{2}-7\right) & 0 \\
\left(8 z_{2}-7\right) z_{2} & -1
\end{array}\right] \\
& \tilde{N}_{2}\left(z_{2}\right)=\left[\begin{array}{cc}
\frac{1}{5}\left(8 z_{2}-7\right) z_{2} & 1 \\
-\frac{1}{5}\left(8 z_{2}^{3}-7 z_{2}^{2}+25\right) & 0
\end{array}\right]
\end{aligned}
$$

そして, $\operatorname{det} \tilde{D}_{1}(0)=-21 / 16 \neq 0, \operatorname{det} \tilde{D}_{2}(0)=-7 \neq 0$ で あるので，(22)式の $R_{k}, k=1,2$, は

$$
R_{1}=\left[\begin{array}{cc}
0 & -8 \\
-\frac{1}{14} & 0
\end{array}\right], \quad R_{2}=\left[\begin{array}{cc}
0 & 0 \\
-\frac{1}{14} & 0
\end{array}\right]
$$

と設定できる。これらの結果を(18)式に代入すると，

$$
\begin{aligned}
& \bar{X}_{1}\left(z_{1}\right)=\left[\begin{array}{cc}
4\left(z_{1}-2\right) & 0 \\
0 & -\frac{1}{28}\left(3 z_{1}+2\right)
\end{array}\right] \\
& \bar{Y}_{1}\left(z_{1}\right)=\left[\begin{array}{cc}
0 & -4 z_{1} \\
\frac{9}{28} z_{1} & 0
\end{array}\right] \\
& \bar{X}_{2}\left(z_{2}\right)=\left[\begin{array}{cc}
\frac{4}{5}\left(2 z_{2}^{3}-z_{2}^{2}+4\right) & 0 \\
-\frac{4}{35} z_{2}^{2} & -\frac{1}{14}
\end{array}\right] \\
& \bar{Y}_{2}\left(z_{2}\right)=\left[\begin{array}{cc}
4 z_{2}\left(2 z_{2}-1\right) & 0 \\
-\frac{4}{7} z_{2} & 0
\end{array}\right]
\end{aligned}
$$

が得られる。

$\operatorname{det} \bar{X}_{1}(0)=4 / 7 \neq 0, \operatorname{det} \bar{X}_{2}(0)=-8 / 35 \neq 0$ であるから, $\bar{X}_{1}^{-1}(0)$ と $\bar{X}_{2}^{-1}(0)$ が存在する。したがって, 方程式 (27) の解はつぎのように求められる.

$$
X_{1}\left(z_{1}\right)=\left[\begin{array}{cc}
\frac{1}{2}\left(2-z_{1}\right) & 0 \\
0 & \frac{1}{2}\left(3 z_{1}+2\right)
\end{array}\right]
$$

$$
\begin{aligned}
& Y_{1}\left(z_{1}\right)=\left[\begin{array}{cc}
0 & \frac{1}{2} z_{1} \\
-\frac{9}{2} z_{1} & 0
\end{array}\right] \\
& \Phi_{1}\left(z_{1}\right)=\left[\begin{array}{cc}
-\frac{1}{16}\left(2 z_{1}-5\right) & 0 \\
0 & -14
\end{array}\right] \\
& X_{2}\left(z_{2}\right)=\left[\begin{array}{cc}
\frac{1}{4}\left(2 z_{2}^{3}-z_{2}^{2}+4\right) & 0 \\
\frac{8}{5} z_{2}^{2} & 1
\end{array}\right] \\
& Y_{2}\left(z_{2}\right)=\left[\begin{array}{cc}
\frac{5}{4} z_{2}\left(2 z_{2}-1\right) & 0 \\
8 z_{2} & 0
\end{array}\right] \\
& \Phi_{2}\left(z_{2}\right)=\left[\begin{array}{cc}
\frac{5}{16} & 0 \\
0 & -14
\end{array}\right]
\end{aligned}
$$

明らかに， $X_{1}(0)=I, X_{2}(0)=I$ なので，(28)式より，

$$
\begin{aligned}
& X_{1}^{\prime}\left(z_{1}\right)=X_{1}\left(z_{1}\right)-I=\left[\begin{array}{cc}
-\frac{1}{2} z_{1} & 0 \\
0 & \frac{3}{2} z_{1}
\end{array}\right] \\
& X_{2}^{\prime}\left(z_{2}\right)=X_{2}\left(z_{2}\right)-I=\left[\begin{array}{cc}
\frac{1}{4}\left(2 z_{2}-1\right) z_{2}^{2} & 0 \\
\frac{8}{5} z_{2}^{2} & 0
\end{array}\right]
\end{aligned}
$$

しかも, $X_{1}^{\prime}(0)=0, X_{2}^{\prime}(0)=0$.

(34)，(35)式より，方程式(14)の解は

$$
X\left(z_{1}, z_{2}\right)=X_{1}^{\prime}\left(z_{1}\right)+X_{2}^{\prime}\left(z_{2}\right)+I
$$

$$
=\left[\begin{array}{cc}
-\frac{1}{4}\left(2 z_{1}-2 z_{2}^{3}+z_{2}^{2}-4\right) & 0 \\
\frac{8}{5} z_{2}^{2} & \frac{1}{2}\left(3 z_{1}+2\right)
\end{array}\right]
$$

$Y\left(z_{1}, z_{2}\right)=Y_{1}\left(z_{1}\right)+Y_{2}\left(z_{2}\right)$

$$
=\left[\begin{array}{cc}
\frac{5}{4} z_{2}\left(2 z_{2}-1\right) & \frac{1}{2} z_{1} \\
-\frac{1}{2}\left(9 z_{1}-16 z_{2}\right) & 0
\end{array}\right]
$$

と構成される。また，(36)式より， $\Phi\left(z_{1}, z_{2}\right)$ は

$$
\Phi\left(z_{1}, z_{2}\right)=\left[\begin{array}{ll}
\Phi_{11}\left(z_{1}, z_{2}\right) & \Phi_{12}\left(z_{1}, z_{2}\right) \\
\Phi_{21}\left(z_{1}, z_{2}\right) & \Phi_{22}\left(z_{1}, z_{2}\right)
\end{array}\right]
$$

である、ただし，

$$
\begin{gathered}
\Phi_{11}\left(z_{1}, z_{2}\right)=\frac{4 z_{2}^{3} z_{1}^{2}-2 z_{2}^{3} z_{1}-2 z_{2}^{2} z_{1}^{2}+z_{2}^{2} z_{1}-4 z_{1}+10}{32} \\
\Phi_{12}\left(z_{1}, z_{2}\right)=-\frac{z_{1} z_{2}\left(4 z_{2}^{4}-4 z_{2}^{3}+z_{2}^{2}-40 z_{2}+8\right)}{4} \\
\Phi_{21}\left(z_{1}, z_{2}\right)=\frac{z_{1} z_{2}\left(2 z_{1}-1\right)\left(32 z_{2}-45\right)}{160} \\
\Phi_{22}\left(z_{1}, z_{2}\right)=-\frac{z_{1} z_{2}\left(64 z_{2}^{3}-122 z_{2}^{2}+45 z_{2}-1120\right)+280}{20} \\
\text { ここで, } \Phi\left(z_{1}, 0\right)=\Phi_{1}\left(z_{1}\right), \Phi\left(0, z_{2}\right)=\Phi_{2}\left(z_{2}\right) \text { であることが }
\end{gathered}
$$


簡単に確認できる。

最後に, (39) 式より, $U\left(z_{1}, z_{2}\right), V\left(z_{1}, z_{2}\right)$ を求め, 定理 4.2 によってPのPractical 安定化補償器のクラスを構成 すればよい.

〔例 5.2〕例 5.1 で与えられたプラントと設計した閉 ループ系を考える。(55)式の閉ループ系の伝達関数行列 において， $Q=q^{-1}\left(z_{1}, z_{2}\right) I \in \boldsymbol{M}(\boldsymbol{H})$, ただし

$$
q\left(z_{1}, z_{2}\right)=3+z_{1}+z_{2}-1.08 z_{1} z_{2}
$$

とおくと, システムの入力 $u_{1}$ から出力 $y_{2}$ までの伝達関数 行列 $H_{y_{2} u_{1}}$ はつぎのようになっている。

$$
H_{y_{2} u_{1}}=N_{p} Q=q^{-1} N_{p} \triangleq\left[\begin{array}{ll}
h_{11} & h_{12} \\
h_{21} & h_{22}
\end{array}\right]
$$

ただし，簡単のため $h_{12}$ のシミュレーションだけを行う ので，ここでは $h_{12}$ のみを示す。

$$
h_{12}\left(z_{1}, z_{2}\right)=\frac{8 z_{1}-2 z_{2}^{3}+z_{2}^{2}-4}{2\left(3+z_{1}+z_{2}-1.08 z_{1} z_{2}\right)}
$$

文献 2)の方法で, $q\left(z_{1}, z_{2}\right)$ が $\bar{U}^{2}$ に零点をもち, BIBO 安定ではないことが判断できる.ところが, 定理 2.2 に よって, $q\left(z_{1}, z_{2}\right)$ は Practical 安定であることがわかる.

$h_{12}\left(z_{1}, z_{2}\right)$ のインパルス応答についてのシミュレー ション結果を Fig. 2 に示す. Fig. 2 の結果より, インパ ルス応答がおよそ座標平面の対角線に沿って振動してい く様子がわかる，実は，このように振動があればそれが 必ず抢々そ対角線に沿うことは Practical 安定なインパ ルス応答の特徵といえる。つまり，このとき，たとえば $z_{1}$ 軸をある有限の值で切り取った場合, $z_{2}$ 軸の方向にお いて, インパルス応答は, ステップ数が十分大きくなる につれて, 必ず零へ収束する.しかも, $z_{1}$ と $z_{2}$ の位置を 置き換えて考えても成り立つ.しかし， $z_{1}$ と $z_{2}$ が同時に 無限大になる場合を考えるとき，インパルス応答は必ず しも零に収束しない.これは, 従来の BIBO 安定性の観 点からみるとき, このシステムが不安定である結論と矛 盾していないことが明らかである.

また，同じ分母 $q\left(z_{1}, z_{2}\right)$ を用い，ただし分子を

$$
\begin{aligned}
b\left(z_{1}, z_{2}\right)= & 1+2 z_{1}+z_{1}^{2}+2 z_{2}+z_{2}^{2}+4 z_{1} z_{2} \\
& +2 z_{1} z_{2}^{2}+2 z_{1}^{2} z_{2}+z_{1}^{2} z_{2}^{2}
\end{aligned}
$$

とするときの伝達関数 $b\left(z_{1}, z_{2}\right) / q\left(z_{1}, z_{2}\right)$ のインパルス応 答をFig. 3 に示す.この結果から, 従来の BIBO安定性を 満たさないが, かなりよい動的性能をもつ Practical 安定なシステムが存在することが確認できる。これは文 献 7)で指摘された $2 \mathrm{D}$ システムのインパルス応答が零に 収束してもそのシステムが必ずしも BIBO 安定となら ないことを示しているとも思われる.この点では, 1Dの 場合と大きく違うので, 注意が必要である.また, Fig. 3 の結果を Fig. 2 と比べてみると, システム伝達関数の零 点は，そのシステムの動的性能に対して相当の影響を与

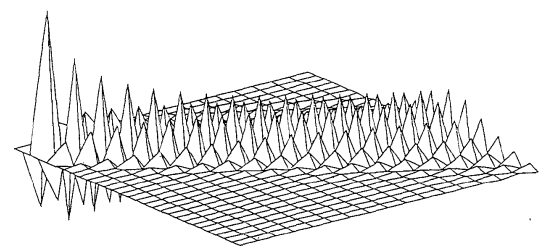

Fig. 2 Impulse response of $h_{12}\left(z_{1}, z_{2}\right)$

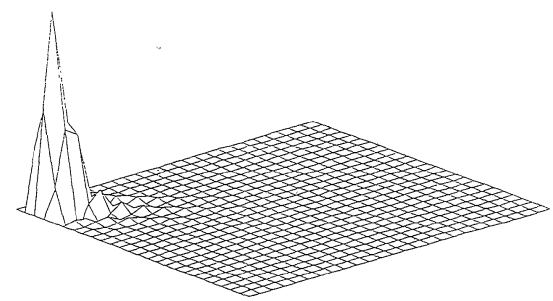

Fig. 3 Impulse response of $b\left(z_{1}, z_{2}\right) / q\left(z_{1}, z_{2}\right)$

えていることもわかる，言い換えれば，満足な動的性能 をもつ $n \mathrm{D}$ システムを設計するには，(Practical または 従来の BIBO）安定化だけではまだ不十分であるといえ よう。

\section{6. 結 び}

本論文では, Agathoklis氏らによって導入された Practical-BIBO 安定の概念に基づいて， $n \mathrm{D}$ システムの フィードバック Practical 安定化問題を考えた. 特に, 従 来の BIBO 可安定ではないが Practical 可安定である $n \mathrm{D}$ システムに重点をおいて考察を行った.

最近，学習制御系や multipass プロセスなどを $2 \mathrm{D} シ$ ステムモデルでとらえ，それに $2 \mathrm{D}$ システムの理論を適 用し解析するといった， $n \mathrm{D}$ システム理論の応用として 興味深い報告がなされている ${ }^{14) ~ 16)}$. これらのシステムに みられる共通の特徵としては, 繰り返す回数に特に制限 はないが，繰り返されるプロセスは有限時間区間に制限 されている、したがって，本論文の結果をもとに，これ らのシステムに対する統一的な設計法を構成することも 可能であろうと考えている.

また，状態空間における $n \mathrm{D}$ システムの Practical 安 定化補償器設計抢よびそれと本論文の代数法との関連に ついても考察を行ったが，つぎの機会に報告する.

\section{参 考 文 献}

1) P. Agathoklis and L. Bruton: Practical-BIBO Stability of n-Dimensional Discrete Systems, IEE Proc. G. Electron. Circuits \& Systems, 6, 236/242 (1983)

2) T.S. Huang: Stability of Two-Dimensional Recursive Filters, IEEE Trans., AU-20, 158/163 (1972)

3) T. Kaczorek, Two-Dimensional Linear Systems, Ber- 


\section{2 年 9 月}

lin: Springer-Verlag, p. 127 (1985)

4) J.P. Guiver and N. K. Bose: Causal and Weakly Causal 2-D filters with Applications in Stabilization, Multidimensional Systems Theory, (ed. N.K. Bose) Dordrecht: Reidel, p. 52 (1985)

5) L. Xu, O. Saito and K. Abe: A Note on Stabilization Algorithms for 2-D Systems, 第 13 回 Dynamical System Theory シンポジウム資料，333/336(1991)

6) E. I. Jury: Stability of multidimensional scalar and matrix polynomials, Proc. IEEE, 66-Sept., 1018/1047 (1978)

7) D. Goodman: Some Stability Properties of TwoDimensional Linear Shift-Invariant Digital Filters, IEEE Trans. Circuits and Systems, CAS-24, 201/208 (1977)

8) M. N. Swamy, L. M. Roytman and E. I. Plotkin: On Stability Properties of Three- and Higher Dimensional Linear Shift-Invariant Digital Filters, IEEE Trans., CAS-32, 888/891 (1985)

9) N.K. Bose: Applied Multidimensional System Theory, Van Nostrand Reingold (1982)

10) V. Kučera : Discrete Linear Control : The Polynomial Equation Approach, Chichester: Wiley (1979)

11) M. Vidyasagar, H. Schneider and B. A. Francis: Algebraic and Topological Aspects of Feedback Stabilization, IEEE Trans., AC-27-4, 880/894 (1982)

12) M. Vidyasagar: Control System Synthesis: A Factorization Approach, Cambridge, MA : MIT Press (1985)

13) C. A. Desoer, R. Liu, J. Murray and R. Saeks: Feedback Systems Design: The Fractional Representation $\Lambda$ pproach to Analysis and Synthesis, IEEE Trans., AC $-25,399 / 412(1980)$

14) F. M. Boland and D. H. Owens: Linear Multipasses: A Two-Dimensional Interpretation, IEE Proc. 127, Pt. D-5, 189/193 (1980)

15) Z.Geng, R.Carroll and J. Xie: Two-Dimensional Model and Algorithm Analysis for a Class of Iterative Learning Control Systems, Int. J. Control, 52-4, 833/862 (1990)

16）宮里, 大島：学習制御系の一設計法, 計測自動制御学会論 文集, 23-6,576/583 (1987)
第 28 巻 第 9 号

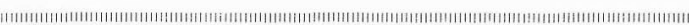

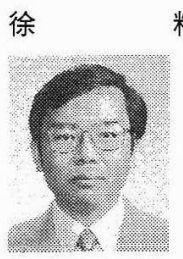

粒 (学生会員)

1982 年, 中国華中理工大学自動制御系卒 業. 90 年豊橋技術科学大学大学院工学研究 科情報工学専攻修士課程修了, 同年同大学 大学院システム情報工学専攻博士後期課程 入学, 現在に至る. 多次元線形システム理 論，および計算機代数学の多次元制御理論 への応用の研究に従事.

池田 貴 俊 (学生会員)

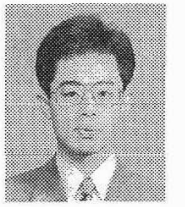

1992 年, 豊橋技術科学大学大学院工学研 究科情報工学専攻修士課程修了, 同年特許 庁入庁, 現在に至る。在学中, 多次元線形 システムの安定性に関する研究に従事.

\section{斉 藤 制 海（正会員）}

(Vol. 28, No. 2 参照)

阿 部 健 一 (正会員)

(Vol. 28, No. 2 参照)

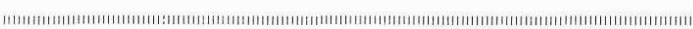

